\title{
Ejercicio físico y síndrome premenstrual
}

\section{Physical exercise and premenstrual syndrome}

\section{Cristóbal Luque García, Ainara Bernal García ${ }^{1}$ \\ ${ }^{1}$ Facultad Ciencias del Deporte. Universidad Pablo de Olavide, Sevilla, España}

\section{Resumen}

El síndrome premenstrual es una enfermedad que afecta al 20-30\% de la población mundial femenina que se encuentra en edad menstrual. Entre su sintomatología se halla una gran variedad de afectaciones de las capacidades físicas, cognitivas y sociales. La actividad física es considerada como un tratamiento no farmacológico para esta enfermedad. El objetivo de este trabajo es elaborar una propuesta de intervención de ejercicio físico para el tratamiento del síndrome premenstrual en mujeres jóvenes en base a una revisión bibliográfica no sistemática. Existe evidencia científica en materia de ejercicio físico y síndrome premenstrual que determina la dosis y la modalidad de ejercicio que producen mejoras en la sintomatología de la enfermedad. El ejercicio del Baduanjin, el yoga y el ejercicio aeróbico son modalidades que producen mejoras y que, por tanto, deberían incluirse como tratamiento. A partir de los resultados obtenidos, se plantea una propuesta de intervención basada en el Baduanjin, yoga y ejercicio aeróbico
Fecha de recepción:

18-08-2020

Fecha de aceptación:

$31-12-2020$

\section{Palabras clave}

Síndrome premenstrual; jóvenes; Baduanjin; yoga; ejercicio aeróbico.

Keywords

Premenstrual syndrome; young women; Baduanjin; yoga; aerobic exercise.

\section{Abstract}

Premenstrual syndrome is a disease that affects $20-30 \%$ of the female world population in menstrual age. Among its symptoms are a wide variety of physical, cognitive and social affectations. Physical activity is recognized as a non-pharmacological treatment for this disease. The main aim of this project is to develop a proposal of physical exercise intervention for the treatment of premenstrual syndrome in young women based on a non-systematic review. There is scientific evidence on physical exercise and premenstrual syndrome that determines the dose and modality of exercise that improves the symptomatology of the disease. Baduanjin exercise, yoga and aerobic exercise are modalities that reduces the burden of the disease and, therefore, should be included as treatment. Based on the result obtained, this project develops an intervention proposal based on Baduanjin, yoga and aerobic exercise. 


\section{Introducción}

Según Qiao et al. (2012), la prevalencia del síndrome premenstrual (PMS) oscila entre el 2030\% de la población mundial femenina que presenta menstruación.

Dentro de sus posibles tratamientos se encuentra el enfoque no farmacológico, el cual hace referencia a la adquisición de estilos de vida saludables. En esta línea, autores como Yonkers y Simoni (2017) afirmaron que el ejercicio físico se podría considerar un tratamiento basado en la evidencia científica; Tsai, Kuo, Kuo y Liao (2018) asociaron el ejercicio físico regular a una disminución de la sintomatología asociada al síndrome premenstrual; Mohebbi Dehnavi, Jafarnejad y Sadeghi Goghary (2018) reportaron beneficios del ejercicio aeróbico en el tratamiento de la sintomatología del PMS; y Zhang, Zhu, Song y Kong (2015) Ilegaron a la conclusión de que el ejercicio del Baduanjin mejoraba los síntomas físicos y mentales del PMS.

No obstante, Kroll-Desrosiers et al. (2017), concluyeron que no existían evidencias significativas que asociaran "actividad física" y "síndrome premenstrual".

Por el contrario, Teixeira, Oliveira y Dias (2013) determinaron que existía una influencia negativa entre el nivel de actividad física y la incidencia del PMS.

La falta de consenso en la bibliografía consultada fundamenta la necesidad de seguir profundizando en el estudio de estas dos variables.

El objetivo general de este artículo es el de elaborar una propuesta de intervención de ejercicio físico para el tratamiento del síndrome premenstrual en mujeres jóvenes en base a una revisión no sistemática.

Los objetivos específicos se concretan en:

- Definir qué es el síndrome premenstrual.

- Distinguir cuáles son los tratamientos que se usan actualmente para paliar su sintomatología.

- Analizar los distintos programas de intervención basados en el ejercicio físico que se pueden llevar a cabo, así como sus características más importantes, como el tipo de ejercicio o la duración.

- Definir las características adecuadas de un programa de intervención basado en el ejercicio físico para mujeres jóvenes con síndrome premenstrual. 


\section{Síndrome premenstrual}

\subsection{Antecedentes}

Una de las primeras evidencias científicas que se poseen con relación al síndrome premenstrual llegaron a principios de los años 30 de la mano del doctor Robert T. Frank. Este científico centró su interés en estudiar las causas hormonales que provocaban un trastorno que él denominó como "tensión premenstrual". Frank (1931) definió este trastorno como la tensión que experimentaban algunas mujeres durante los días previos a su menstruación.

También cabe destacar la aportación que hicieron Greene y Dalton (1953), los cuales pensaron que, en relación con el término que acuñó el doctor Robert, los síntomas que se presentaban antes de la aparición de la menstruación eran mucho más amplios y abstractos que una simple tensión; proporcionando entonces la nomenclatura de síndrome premenstrual.

Así pues, en la búsqueda de una definición y unos criterios de diagnóstico más claros y concisos, O'Brien et al. (2011) crearon un debate proporcionando un enfoque coherente que sirviera a clínicos e investigadores.

Por último, y solo dos años más tarde, la Asociación Americana de Psiquiatría (APA) publicó el libro Diagnostic And Statiscal Manual Of Mental Disorders (DSM-V). En este libro se recogían los criterios de diagnóstico de una gran variedad de trastornos mentales, entre los que se incluyeron los del Trastorno Disfórico Premenstrual (PMDD), definiendo los criterios de diagnóstico, sintomatología y posibles variables patológicas, entre las cuales se encuentra el Síndrome Premenstrual (PMS).

Por tanto, es necesario conocer qué es el PMDD, puesto que el PMS se encuentra actualmente recogido como una variedad en el diagnóstico del PMDD.

\subsection{Diagnóstico}

En este apartado vamos a explicar cuáles son los criterios y la variedad de diagnóstico del PMDD, según recoge la Asociación Americana de Psiquiatría (APA) en su manual Diagnostic And Statiscal Manual Of Mental Disorders (DSM-V).

\subsubsection{Criterios de diagnóstico:}

El diagnóstico del PMDD viene definido por los siguientes criterios: 
El primero (A) detalla que las mujeres deben presentar una serie de síntomas la semana previa al comienzo de su menstruación. Moderándose estos en los días cercanos a la menstruación y descendiendo en intensidad o desapareciendo en las semanas siguientes.

El segundo (B) redacta que uno o más de los síntomas que deben estar presentes son:

- Gran labilidad afectiva (como cambios de ánimo, sentirse de repente mal o con ganas de llorar, o aumento de la sensibilidad al rechazo)

- Gran irritabilidad, enfado o aumento de los problemas interpersonales.

- Gran estado de depresión, sentimientos desesperanzados, o pensamientos de autodesprecio.

- Gran ansiedad, tensión, y/o sentimiento de no encajar.

El tercero (C) redacta que uno o más de los siguientes síntomas también deben de estar presentes:

1. Descenso del interés por las actividades cotidianas.

2. Dificultad subjetiva de concentración.

3. Letargo o ausencia de energía.

4. Gran cambio de apetito; comer en exceso o ansias de comida.

5. Hipersomnio o insomnio.

6. Sensación de perder el control.

7. Síntomas físicos como sensibilidad en los senos, hinchazón, dolor en músculos y articulaciones, ganancia de peso.

Cabe destacar que los síntomas de los criterios A-C se deben haber venido repitiendo en la mayoría de los últimos doce ciclos menstruales.

El cuarto (D) asocia los síntomas con una angustia clínicamente significativa o con una interferencia con las actividades sociales cotidianas, trabajo o colegio. (por ejemplo, evitación de las actividades sociales, descenso de la productividad o eficiencia en el trabajo, casa o el colegio).

El quinto (E) concreta que la perturbación no se atribuye a los síntomas de otro trastorno, como un trastorno depresivo mayor, pánico, trastorno depresivo persistente, o un trastorno de la personalidad. (aunque si es posible que pueda coocurrir con alguno de ellos).

El sexto (F) determina que el criterio A debe estar confirmado por una evaluación diaria prospectiva durante al menos dos ciclos sintomáticos. 
El séptimo (G) concreta que los síntomas no son atribuibles a los efectos psicológicos de una sustancia (como el abuso de las drogas o los fármacos) o alguna otra condición médica (hipertiroidismo).

Definidos todos los criterios, cabe destacar que para que se diagnostique el PMDD, debe de darse la característica de que al menos cinco de los síntomas que hemos citado deben estar presentes. De no ser así, la seguiría un proceso diagnóstico diferente, el cual veremos a continuación.

\subsubsection{Variedad en el diagnóstico:}

Síndrome Premenstrual: Este diagnóstico difiere del PMDD en que no es necesario un mínimo de cinco síntomas; además, puede no existir una alteración en el estado afectivo del sujeto. Esta característica puede ser la razón de que el PMS sea más común que el PMDD. Por otro lado, aunque ambos comparten la mayoría de la sintomatología, se considera al PMS menos severo que al PMDD. Cabe destacar que la presencia de síntomas físicos o comportamentales en el periodo previo a la menstruación probablemente se deban al PMS y no al PMDD, aunque la persona no presente síntomas afectivos.

Dismenorrea: La dismenorrea se caracteriza por producir menstruaciones dolorosas, pero no es una patología que presente cambios afectivos. Suele presentarse al inicio de la menstruación, lo que la diferencia del PMDD, que se presentaba antes del comienzo de la menstruación.

Trastorno bipolar, trastorno depresivo mayor y trastorno depresivo persistente (distimia): Para el diagnóstico de esta patología es necesario que esta sea confirmada por la evaluación diaria prospectiva elaborada por Rubinow (1984). Esto se debe a que, en muchos casos, la sintomatología que presentan muchas de las mujeres tiene que ver con los trastornos bipolar y depresivo, y no con el PMDD.

Uso de tratamiento hormonal: El uso de tratamientos hormonales, como es el caso del contraceptivo, pueden dar lugar a síntomas premenstruales moderados o severos. Si estos ocurren al inicio de la ingesta de hormonas, posiblemente se relacione con esta causa y no con el PMDD. Además, si la persona deja de utilizar estas hormonas y los síntomas desaparecen, se confirmará que su trastorno ha estado inducido por el consumo de dichas sustancias y no por el PMDD. 


\subsection{Epidemiología}

Las investigaciones que se llevan a cabo sobre esta población determinada tienen como objetivo el de proporcionar estimaciones sobre la afectación del PMS y PMDD. Pese a ser muy difíciles de realizar, debido a la gran sintomatología asociada y a su evaluación subjetiva, existen varios informes fiables. Tomando como muestra de estudio toda la población mundial femenina en edad menstrual, estos informes han revelado que un 20-30\% de esta población sufre PMS. Por otro lado, la tasa de población que padece PMDD es de un 1.2 - 6.4\% (Yonkers y Simoni, 2017). También cabe destacar que la prevalencia del PMS en las mujeres españolas en edad menstrual es del 2.5-14\% (Del Burgo, Trigueros, Ruiz, Muñiz y Maestre, 2006).

\subsection{Factores de riesgo}

Pilver, Kasl, Desai y Levy (2011) afirmaron que las mujeres blancas muestran más tendencia a padecer PMS y otros trastornos psíquicos que las mujeres afroamericanas. Además, comprobaron que estos trastornos están influenciados por diferencias culturales. Wittchen, Becker, Lieb y Krause (2002) señalaron que el riesgo no es dependiente de la edad en mujeres premenopáusicas.

Por otro lado, Chocano-Bedoya et al. (2013) comprobó que factores como la dieta modulan positivamente el riesgo de sufrir PMS. La alta ingesta de tiamina, riboflavina, hierro no hemo y zinc se consideró como un método preventivo contra la enfermedad, mientras que un alto consumo de potasio podría, por el contrario, incrementar el riesgo de padecerla.

Bertone-Johnson, Hankinson, Willet, Johnson y Manson (2010) y Hashemi et al. (2016) afirmaron haber encontrado evidencias que sugerían que la adiposidad y el síndrome metabólico aumentaba el riesgo de sufrir PMS, en concreto en mujeres de más de 25\%kg/m2.

Otros factores de riesgo que se han asociado al PMS son el consumo de cigarrillos con nicotina, según Bertone-Johnson, Hankinson, Willet, Johnson y Manson (2010); los abusos sexuales tempranos y el trauma, tal y como apuntan Perkonigg, Yonkers, Pfister, Lieb y Wittchen (2004) y Bertone-Johnson, Whitcomb, Missmer, Manson, Hankinson y Rich-Edwards (2014).

También se ha confirmado que la comorbilidad entre los trastornos depresivos y/o de ansiedad y el PMS es alto, tal y como señala Graze, Nee y Endicott (1990). 
Por último, Kendler, Silberg, Neale, Kessler, Heath y Eaves (1992) y Condon (1993) afirmaron en sus diferentes estudios que los gemelos monocigóticos tenían más posibilidades de sufrir PMS que los dicigóticos.

\subsection{Principales adaptaciones fisiológicas del PMS}

Una vez se ha definido la sintomatología asociada al PMS, su epidemiología y sus factores de riesgo, se van a nombrar las principales adaptaciones fisiológicas que se han encontrado en pacientes con esta patología.

Aunque la investigación no apoya que la enfermedad se deba únicamente a un exceso o déficit simple de una hormona, parece ser que existe relación entre los cambios hormonales que presentan las mujeres y la expresión de los síntomas de esta patología (Schmidt et al., 2017). Así bien, la disminución de los niveles de alopregnanolona, metabolito de la progesterona, ayuda a paliar los síntomas premenstruales (Martinez et al., 2015). Además, un aumento de los inhibidores de la recaptación de la serotonina (ISRS), también mitiga los síntomas asociados al PMS (Gracia et al., 2009).

A modo de resumen, la figura 1 muestra las principales adaptaciones:

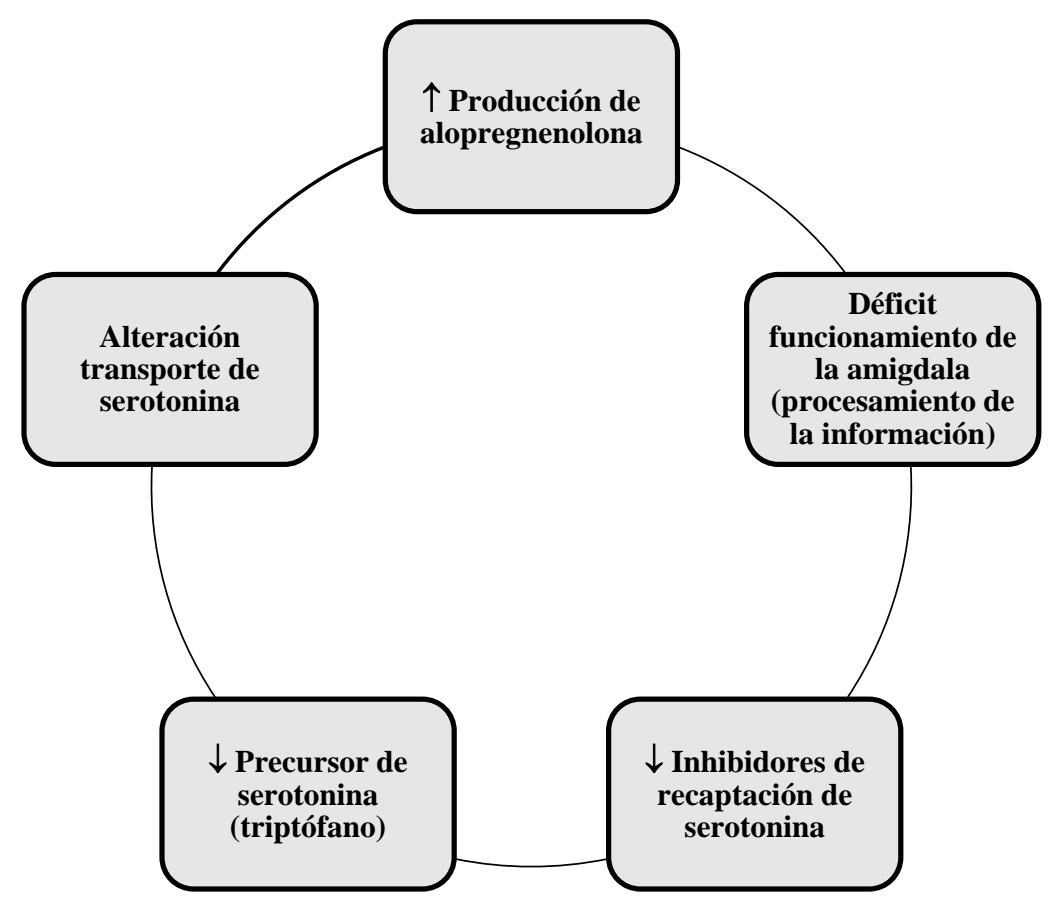

Figura 1. Principales adaptaciones fisiológicas del PMS. Fuente: Elaboración propia. 


\section{Tratamiento}

\subsection{Perspectivas de tratamiento del PMS}

El tratamiento del PMS puede abarcarse desde 4 grandes perspectivas (Yonkers y Simoni, 2017):

1. Enfoque no farmacológico, como dieta, ejercicio físico y psicoterapia.

2. Tratamiento psicotrópico.

3. Agonistas y antagonistas hormonales.

4. Vitaminas y botánica.

Con relación al enfoque no farmacológico, la mayor parte de las recomendaciones de este apartado hacen referencia a la adquisición de hábitos de vida saludables. La realización de ejercicio físico, una dieta alta en carbohidratos complejos o la Terapia Cognitiva Conductual (TCC) pueden ser beneficiosas en la reducción de la sintomatología del PMS (Yonkers y Simoni, 2017).

El tratamiento psicotrópico más conocido y respaldado por la evidencia científica es el de los Inhibidores de Recaptación de Serotonina (IRS), tal y como afirman Marjoribanks, Brown, O'Brien y Wyatt (2013).

Desde la perspectiva agonistas y antagonistas hormonales existe escasa evidencia científica que apoye el uso de los anticonceptivos orales combinados como tratamiento para el PMS (Yonkers y Simoni, 2017). No obstante, algunos ensayos como el de Pearlstein, Bachmann, Zacur y Yonkers (2005) aseguran que existen combinaciones de anticonceptivos orales que ayudan a paliar la sintomatología del PMDDy, en consecuencia, del PMS.

También se ha buscado un tratamiento en el uso de estrógeno (Naheed, Kuiper, Uthman, O'Mahony y O'Brien, 2017) y en la progesterona, pero las investigaciones que respaldan dichos tratamientos son consideradas de baja calidad (Ford, Lethaby, Roberts y Mol, 2009).

Parece ser que los agonistas de las hormonas que liberan gonadotropinas ( $G n R H$ ) utilizados de forma continua, suprimen la liberación de estrógenos y la ovulación, lo que disminuye la sintomatología del PMS (Wyatt, Dimmock, Ismail, Jones y O'Brien, 2004). No obstante, los efectos secundarios y el alto costo de este tratamiento hacen que su uso se restrinja a casos muy agudos de PMDD o PMS (Yonkers y Simoni, 2017). 
En cuanto al uso de las vitaminas y las plantas como terapia contra la sintomatología del PMS, se ha estudiado que la vitamina B-6 (piridoxina), Vitex Agnus Castus (Chasteberry), la hierba de San Juan, Ginko Biloba y el aceite de Onagra son las más frecuentes (Yonkers y Simoni, 2017).

\subsection{Enfoque no farmacológico: Ejercicio físico}

Tras definir qué es el PMS, cuál es su sintomatología asociada y cuáles son los diferentes tratamientos que, según la evidencia científica, se utilizan para paliar dicha sintomatología, vamos a tratar con más profundidad uno de ellos: el ejercicio físico.

Según Ramírez, Vinaccia y Ramón (2004) existe una gran y creciente evidencia científica que afirma que el ejercicio físico tiene una gran influencia en factores como: "estados emocionales como ansiedad y depresión, disminución del estrés, mejoras de las capacidades intelectuales y cognitivas" ( $p$ 73). Es por esto por lo que debería considerarse a la actividad física como un elemento fundamental en los programas de la promoción de la salud en poblaciones infantojuveniles, ya presenten o no alguna patología (Ramírez et al., 2004).

El PMS se encuentra actualmente recogido, según la $A P A$, como un trastorno mental. Por esta razón, los programas de intervención a través del ejercicio físico no deben de suponer a la persona un aumento del grado de estrés y ansiedad, un aumento del dolor físico ni un deterioro de sus capacidades sociales. Es por ello por lo que, Solís (2013) concluyó que algunos de los ejercicios físicos recomendados como terapia no farmacológica para el PMS son: "Ejercicios aeróbicos, terapias de relajación, ejercicios de respiración, meditación, yoga y masajes terapéuticos." ( $p$ 713). Este tipo de ejercicios, generalmente de poco impacto y cuya ejecución no presenta apenas dificultad, permiten una mejora de las capacidades cognitivas, físicas y sociales. Como complemento a esta recomendación, cabe señalar que algunos de los beneficios de la práctica del yoga para la población general, según Desai, Tailor y Bhatt (2015), son: "aumento de la actividad de las ondas cerebrales alfa, lo que se asocia con mejoras en el estado de ánimo, estado de paz y relajación, liberación de serotonina y creatividad" ( $p$ 7). Esto produce que las personas que practican yoga vean mejorada sus capacidades cognitiva y social, además de la capacidad física.

Otros autores como El-Lithy, El-Mazny, Sabbour y El-Deeb (2015) y Mohebbi Dehnavi Jafarnejad y Sadeghi Goghary (2018) han concluido que la práctica de ejercicio aeróbico de forma regular supone un descenso significativo de algunos de los síntomas cognitivos y físicos que se asocian al PMS. 
Así pues, se ha demostrado que aquellas mujeres que practican deporte de forma regular muestran menos alteraciones del estado de ánimo, la concentración o el afecto antes y durante la menstruación (Delara, Ghofranipour, Azadfallah, Tavafian, Kazemnejad y Montazeri, 2012). Además, el ejercicio regular también se asoció con una disminución de las probabilidades de sufrir dolor menstrual (Tsai et al., 2018).

No obstante y a pesar del gran cuerpo de evidencia científica que respalda el ejercicio físico como tratamiento para el PMS, siempre y cuando este se adapte a las características de la patología, existen estudios como el de Kroll-Desrosiers et al. (2017) que no apoyan que exista relación entre ejercicio físico y descenso en la frecuencia del PMS o en la gravedad de la sintomatología asociada al PMS; si no que la mejoría en la sintomatología se debe principalmente a la adquisición de hábitos de estilo de vida saludables asociados a la alimentación y a terapias farmacológicas.

Como ya se argumentó en la introducción, esta falta de consenso en la bibliografía científica es la que ha motivado la realización del análisis que se presenta a continuación.

\section{Método}

La revisión llevada a cabo en este trabajo se define, según Guirao-Goris, Olmedo y Ferrer (2008) como: "tipo de artículo científico que sin ser original recopila la información más relevante sobre un tema específico" (p 3).

Para determinar el papel que juega el ejercicio físico en la sintomatología del síndrome premenstrual, se analizaron artículos que estudian la relación que existe entre ambas variables: ejercicio físico y patología; en una población concreta: mujeres jóvenes.

Para este trabajo, se ha llevado a cabo una revisión cuyos pasos han sido:

1. Establecimiento de un enunciado para el problema.

2. Determinación de descriptores, motores y estrategias de búsqueda.

3. Búsqueda en fuentes primarias.

4. Lectura, reflexión y elección de la información más relevante.

Para el presente trabajo y atendiendo a sus objetivos, se utilizaron las siguientes fuentes de búsqueda de información: PubMed y LILACS.

Los descriptores o palabras clave que se eligieron para este trabajo fueron:

- PubMed: "Premenstrual Syndrome" AND "exercise" AND "young adult". 
- LILACS: "Premenstrual Syndrome" AND "exercise" AND "young adult".

La búsqueda se limitó según su fecha de publicación al periodo comprendido entre 2009 y 2019 con el objetivo de obtener información lo más actualizada posible.

Además, una de las estrategias que se siguió fue la búsqueda de terminología en inglés, debido a que gran parte de la bibliografía científica se redacta en este idioma.

Una vez obtenidos los resultados de ambas búsquedas, comenzó un proceso de selección de la información. En este se llevó a cabo una lectura de los títulos y los resúmenes de los resultados, a fin de determinar su relevancia en función del objeto de estudio del trabajo. Tras este proceso, y después de eliminar los resultados duplicados, se procedió a eliminar aquellos que no cumplieran con los criterios de inclusión que se muestran en la tabla 1.

Tabla 1. Criterios de inclusión y exclusión. Fuente: Elaboración propia.

\section{Criterios de inclusión}

- Disponibilidad del texto completo

- Población clínica: Mujeres con PMS

- Edad de la población: Menores de 40 años

- Resultados que incluyan efectos de la actividad física sobre diversos parámetros relacionados con el PMS

- Resultados que incluyan programas de intervención basados en el ejercicio físico

\section{Criterios de exclusión}

- Todos los resultados que no cumplan los criterios de inclusión

Tras haber realizado dicho sesgado, se obtuvieron un total de 5 resultados válidos para la búsqueda en PubMed, y ningún resultado válido para la búsqueda en LILACS. 
4.5. Diagrama de flujo

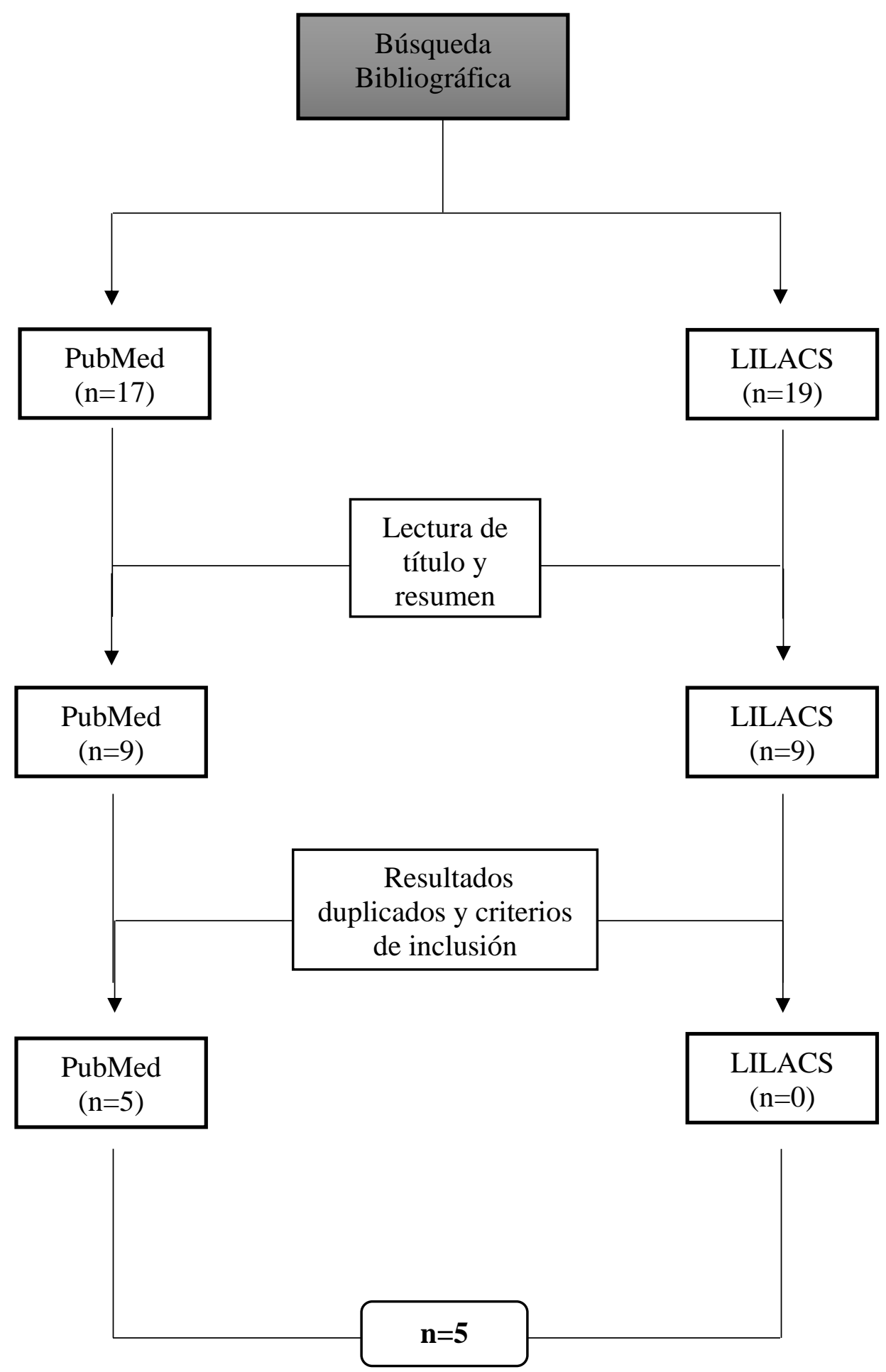

Figura 2. Estrategia de búsqueda y criterios de selección. Fuente: Elaboración propia. 


\section{Resultados}

Para analizar los distintos programas de intervención basados en el ejercicio físico que se pueden llevar a cabo en función de las características específicas de nuestra población de estudio, mujeres jóvenes, se incluyeron en esta revisión un total de 5 artículos. Por un lado, el número total de mujeres que llevaron a cabo los distintos programas de intervención fue de 224, incluyendo aquellas que estuvieron en un grupo Control y las que estuvieron en un grupo Experimental. Por otro, la media de edad de las mujeres fue de 23.35 años.

Para determinar los efectos de los distintos programas en la sintomatología asociada al PMS se utilizaron diferentes métodos de evaluación, como por ejemplo el SF-36 o el DRSP, así como la utilización de diferentes parámetros hormonales y hematológicos asociados al PMS. La duración de los programas varió entre 6 y 12 semanas, con una frecuencia mínima de 2 días en semana y una máxima de 5 días. En la tabla 2 se han recogido las características más importantes de los artículos que se han incluido en esta revisión.

A continuación, se pretende pues detallar los efectos de estos programas, según diversos parámetros, en la sintomatología asociada al PMS en función del tipo de ejercicio.

\subsection{Baduanjin}

Investigaciones recientes sugieren el ejercicio chino del Baduanjin como un remedio contrastado para paliar la sintomatología física y mental del PMS. Se ha demostrado que este ejercicio, que combina las demandas físicas y las cognitivas, produce numerosas mejoras en ambos estados.

Zhang et al. (2015) comprobaron que la puntuación total del Daily Record of Severity Problems (DRSP) disminuyó de forma significativa tras la realización de la intervención en comparación con la puntuación total recogida antes de realizarla. Que la puntuación total del DSRP bajara en el segundo y tercer mes con respecto al primero sugirió que la mejora en la sintomatología comienza tras el primer mes de realización del Baduanjin. En definitiva, Zhang et al. (2015) afirmaron que el ejercicio del Baduanjin mejora el estado físico y mental de las mujeres durante el periodo premenstrual, además de mejorar el estado psicosomático durante todo el ciclo menstrual. Todo ello, tiene como consecuencia la producción de efectos positivos en la salud mental. 
Tabla 2: Resumen de las características de los estudios incluidos. Fuente: Elaboración propia.

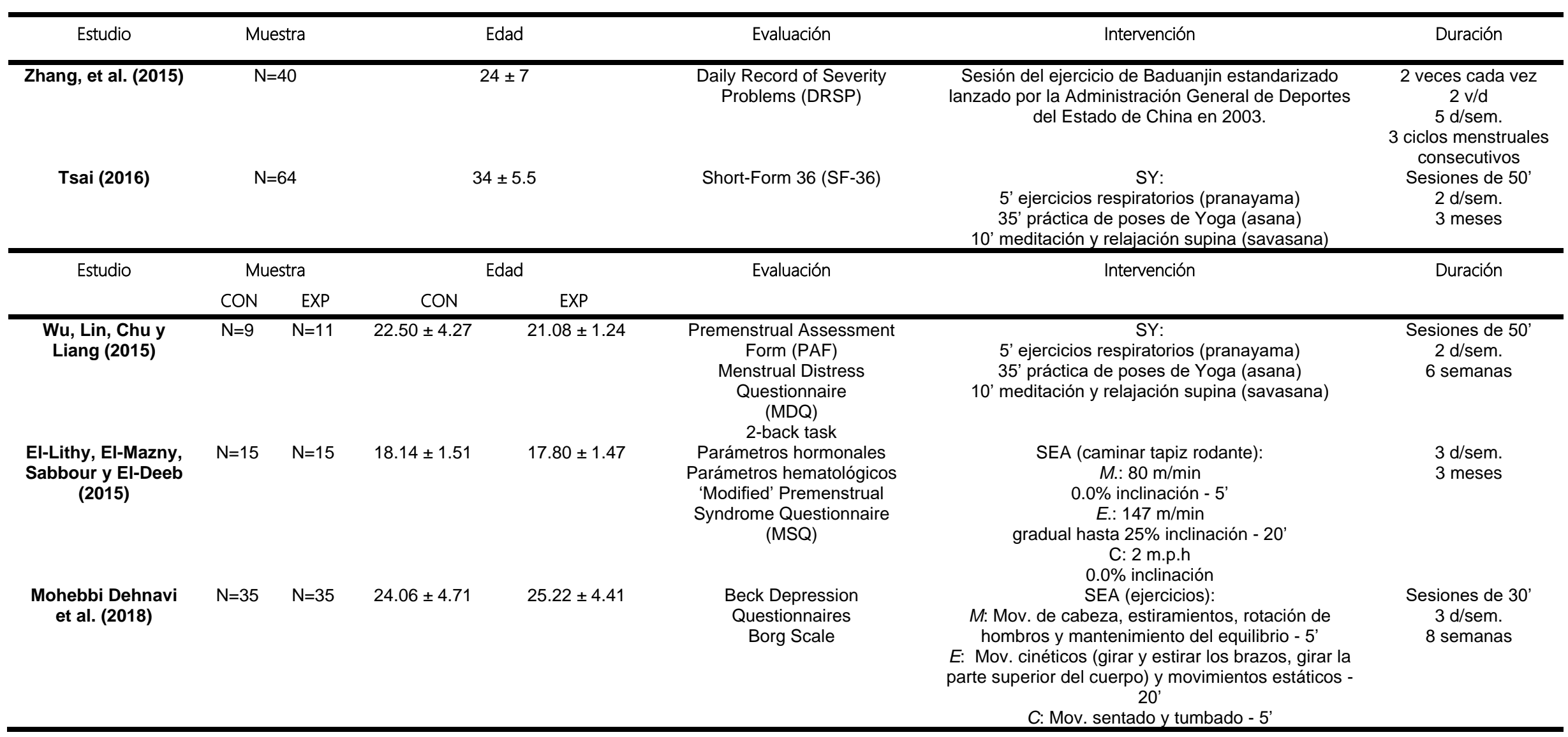

Valores medios \pm SD.

Nota. EXP = Grupo Experimental; CON = Grupo Control; SEA = Sesión de Entrenamiento Aeróbico; SY = Sesión del ejercicio de Yoga; $\mathrm{M}=$ Movilidad y calentamiento; $\mathrm{E}=$ Entrenamiento; $\mathrm{C}=$ Vuelta a la calma; $\mathrm{v} / \mathrm{d}$ = veces al día; $\mathrm{d} / \mathrm{sem}$. = días por semana; Mov = movimientos. 


\subsection{Yoga}

Existe una creciente evidencia científica que propone el ejercicio del yoga como tratamiento para paliar los efectos de esta enfermedad. Este ejercicio, cuya ejecución no presenta apenas dificultad, permite una mejora de las capacidades cognitivas, físicas y sociales.

Tsai (2016) afirmó basándose en los resultados de su programa de intervención que el ejercicio regular de yoga mejoraba las puntuaciones de dolor corporal y función física, variables medidas en el SF-36. Además, varios síntomas auto reportados descendieron de forma significativa tras la intervención. Estos síntomas fueron: inflamación abdominal, sensibilidad en los senos, calambres abdominales y sudores fríos. Es por ello por lo que se considera que el ejercicio regular de yoga disminuye la sintomatología física del PMS. Esta intervención también se relacionó con un descenso del riesgo de subir dolores físicos durante todo el ciclo menstrual.

Wu et al. (2015), que basaron su intervención en medir la producción de ondas cerebrales alfa antes y después de un programa de ejercicios de yoga, comprobaron que tras el ejercicio se elevaba la producción de ondas cerebrales alfa. Como consecuencia, las participantes se sentían más relajadas. Este hallazgo sugiere que las mujeres con PMS que sufren un deterioro de su capacidad cognitiva: depresión, ansiedad, dificultad de concentración, etc., podrían asistir a clases de yoga para mejorar esta vertiente sintomatológica. Wu et al. (2015) concluyeron que: "el ejercicio del yoga puede permitir que las mujeres con PMS movilicen más recursos de atención para concentrarse en una tarea determinada" (p 368).

\subsection{Ejercicio Aeróbico regular}

El-Lithy et al. (2015) reportaron tras su intervención que el ejercicio físico redujo de forma significativa los síntomas premenstruales en mujeres jóvenes. Concretamente, el ejercicio mostró una reducción de síntomas cognitivos como la ansiedad y la depresión durante el periodo premenstrual, además de reducir otros síntomas físicos como calambres menstruales y dolos lumbar durante los primeros días de la menstruación. El-Lithy et al. (2015) concluyeron que:

El-Lithy et al. (2015)

El ejercicio aeróbico tiene efectos muy beneficiosos, tanto fisiológicos como psicológicos, en mujeres jóvenes, pues alivia síntomas premenstruales. Disminuye la prolactina, el estradiol y la progesterona, los cuales están involucrados en la 
fisiopatología del PMS. Además, mejora el hematocrito, hemoglobina y recuento de glóbulos rojos, lo que mejora el bienestar social, emocional y educativo. (p.4)

Los resultados que se reportan de esta investigación son de elevado interés debido a que es el único programa de intervención de los presentes en esta revisión que evalúan variables relacionadas con la fisiopatología del PMS, como por ejemplo la prolactina, el estradiol o la progesterona; además de otros parámetros hormonales y hematológicos que afectan a la capacidad cognitiva y social de las mujeres con PMS.

Mohebbi Dehnavi et al. (2018) reportaron que los ejercicios aeróbicos realizados en su intervención produjeron una disminución significativa de algunos síntomas físicos del PMS como el dolor de cabeza, las náuseas o la diarrea por estreñimiento. Por esta razón, sería aconsejable recomendar ejercicio aeróbico a todas las mujeres en edad reproductiva, y especialmente a aquellas que sufran PMS.

\section{Conclusión}

Como principales conclusiones extraídas de la revisión bibliográfica realizada recogemos que el síndrome premenstrual es una enfermedad mental que se encuentra actualmente clasificada como una variedad en el diagnóstico del trastorno disfórico premenstrual, según la Asociación Americana de Psiquiatría.

Los tratamientos que se usan actualmente para paliar su sintomatología son: Enfoque no farmacológico, tratamiento psicotrópico, agonistas y antagonistas hormonales y vitaminas y botánica.

Los programas de intervención analizados han arrojado que el ejercicio chino del Baduanjin, el ejercicio de yoga y el ejercicio aeróbico regular son aquellos que producen beneficios en los síntomas del síndrome premenstrual en mujeres jóvenes.

La duración de los programas de intervención ha variado entre las 6 y las 12 semanas. Por otro lado, la duración de las sesiones osciló entre los 30 y los 50 minutos, en función de la intensidad del ejercicio (30' ejercicio aeróbico regular y 50' ejercicios de yoga o Baduanjin).

Algunas de las características que debe presentar un programa de intervención basado en el ejercicio físico como tratamiento para mujeres jóvenes con síndrome premenstrual son:

- Tipo de sesiones: Ejercicios de intensidad baja, como por ejemplo andar o practicar yoga. 
- Características de las sesiones: Ambientar las sesiones con música que favorezca estados de relajación y bienestar.

- Realización de las sesiones: Durante todo el ciclo menstrual.

- Duración de las sesiones: 40 minutos.

- Frecuencia de sesiones: 3-4 días/semana.

- Duración del programa: 8-10 semanas.

\section{Propuesta de intervención}

El objetivo general de este artículo es el de elaborar una propuesta de intervención de ejercicio físico para el tratamiento del síndrome premenstrual en mujeres jóvenes en base a una revisión no sistemática. La intervención que se propone a continuación persigue reducir la expresión sintomatológica asociada a esta patología.

Las características generales que presentará la intervención serán las siguientes:

- La duración de la intervención será de 10 semanas.

- La realización de las sesiones será durante todo el ciclo menstrual.

- La frecuencia de las sesiones será de 3 sesiones por semana.

- La duración de las sesiones será de 40 minutos.

- Los ejercicios que se llevarán a cabo serán: Yoga y ejercicio aeróbico.

- El Baduanjin no se incluirá en la propuesta de intervención pues se considera que, debido a las características culturales de la muestra a la que irá dirigida, es un ejercicio poco conocido cuya ejecución puede resultar compleja.

- La intensidad de las clases de yoga se determinará mediante la escala de percepción del esfuerzo de Borg. Se pretende que las clases de yoga sean de nivel suave. Para ello, los sujetos podrían ir variando la ejecución del ejercicio en busca de una RPE aproximada de 10 (número de apoyos, ayudas con bandas elásticas, ayudas con la pared o un compañero, etc.). El objetivo de trabajar con esta intensidad está en la búsqueda del equilibrio entre ejercicio físico y relajación. Es de importancia destacar que esta población suele presentar un deterioro de su capacidad cognitiva.

- Las intensidades del ejercicio aeróbico se determinarán a través del porcentaje de la frecuencia cardíaca máxima (FCmáx) y su relación con la percepción del esfuerzo de Borg. El entrenamiento será modificado en función de la FCmáx. de cada sujeto, buscando que su percepción del esfuerzo sea de 10, es decir, de un nivel suave (aproximadamente 50\% de la FCmáx.). Para ello, será necesario controlar la frecuencia cardíaca durante la realización de la sesión. 
- Para evaluar el resultado de la propuesta de intervención en los sujetos se utilizará, antes y después del programa, el cuestionario SF-36. Este, está constituido por 35 ítems puntuables. Estos ítems se dividen en 8 dimensiones: Función Física, Rol Físico, Rol Emocional, Función Social, Salud Mental, Salud General, Dolor Corporal y Vitalidad.

- El objetivo es el de comparar los resultados del cuestionario antes y después de la ejecución de la propuesta de intervención para determinar su posible efecto sobre las distintas dimensiones de los sujetos, es decir, sobre su salud.

- En la Tabla 3 se ha recogido la distribución general de la propuesta de intervención dentro de una semana.

- En el Anexo 1 se ejemplifica una sesión de yoga válida para esta propuesta de intervención. Cabe añadir que, en función de la RPE de los sujetos, se irá progresando en las sesiones, incluyendo ejercicios de mayor dificultad. 
Tabla 3. Distribución general de la propuesta de intervención elaborada. Fuente: Elaboración propia.

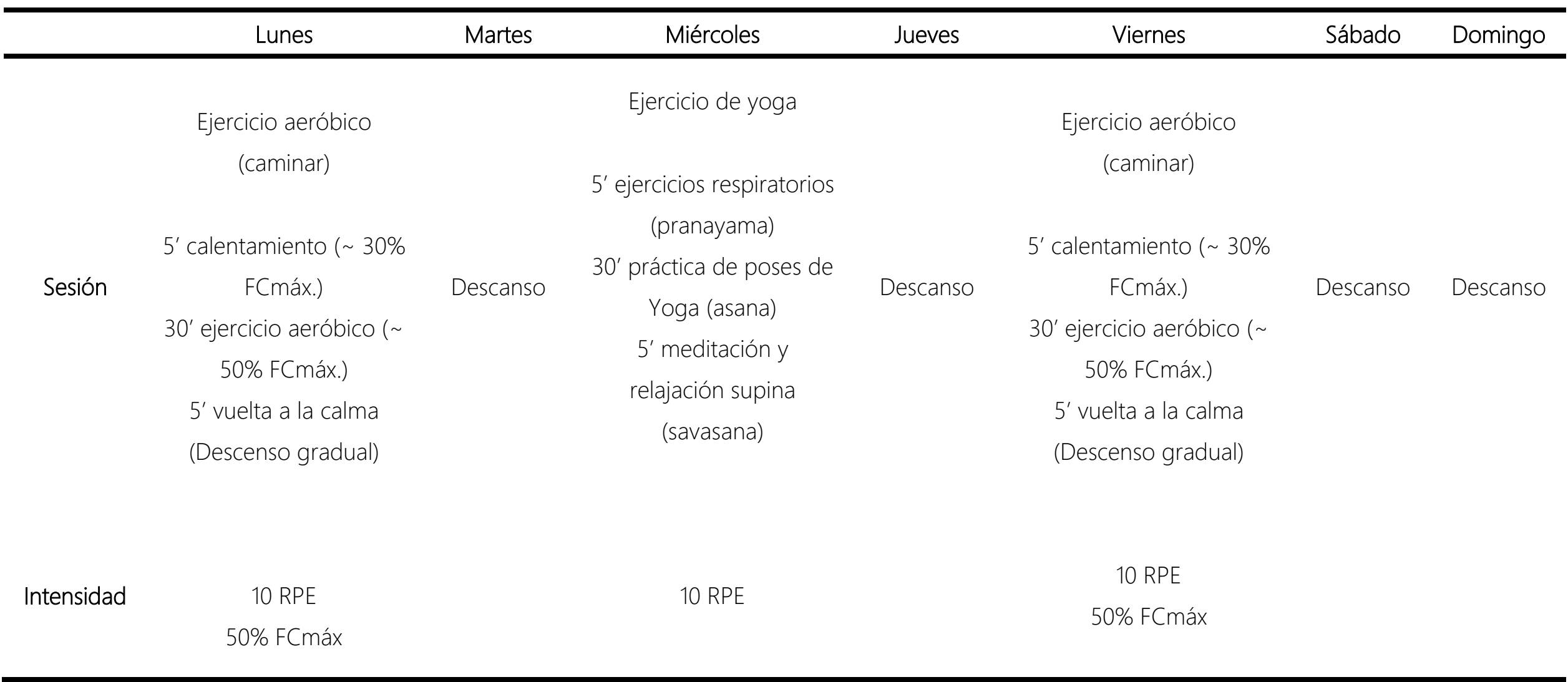




\section{Limitaciones del estudio}

La propuesta de intervención que ha sido descrita en este artículo ha estado condicionada por las siguientes limitaciones:

- El número de artículos que se han revisado y que han servido como base para realizar esta propuesta de intervención han sido escasos. Por lo tanto, es previsible que una revisión de mayor magnitud pudiera aportar mayor precesión a la propuesta realizada.

- Cabe la posibilidad de que los criterios de inclusión y exclusión utilizados hayan sido demasiado restrictivos.

- En el presente trabajo se ha llevado a cabo una propuesta de intervención, pero no se ha puesto en práctica. Como consecuencia, no ha sido posible estudiar los efectos de esta propuesta en la población de estudio.

\section{Futuras líneas de investigación}

Con respecto al tratamiento del síndrome premenstrual a través del ejercicio físico, se abren numerosas líneas de investigación y desarrollo que permitan el avance en este tema. A continuación, se proponen algunas de ellas:

- Realizar una revisión de mayor magnitud que aporte precisión a futuras propuestas de intervención de ejercicio físico para el tratamiento del síndrome premenstrual en mujeres jóvenes.

- Ampliar los criterios de inclusión y las estrategias de búsqueda en función de un mayor rango de edad o del idioma de las investigaciones (español, portugués, etc.)

- Llevar a cabo la propuesta de intervención planteada en el presente trabajo, en busca de unos resultados que nos permitan realizar una valoración de esta.

\section{Conflicto de interés}

Los autores declaran no tener conflicto de interés.

\section{Financiación}

No se ha recibido compensación por la investigación. 


\section{Referencias}

American Psychiatric Association. (2013). Diagnostic and statistical manual of mental disorders (DSM-5®). American Psychiatric Pub. https://www.appi.org/products/dsm-manual-ofmental-disorders

Bertone-Johnson, E. R., Hankinson, S. E., Willett, W. C., Johnson, S. R., y Manson, J. E. (2010). Adiposity and the development of premenstrual syndrome. Journal of women's health, 19(11), 1955-1962.

Bertone-Johnson, E. R., Whitcomb, B. W., Missmer, S. A., Manson, J. E., Hankinson, S. E., y RichEdwards, J. W. (2014). Early life emotional, physical, and sexual abuse and the development of premenstrual syndrome: a longitudinal study. Journal of Women's Health, 23(9), 729-739.

Chocano-Bedoya, P. O., Manson, J. E., Hankinson, S. E., Johnson, S. R., Chasan-Taber, L., Ronnenberg, A. G., ... y Bertone-Johnson, E. R. (2013). Intake of selected minerals and risk of premenstrual syndrome. American journal of epidemiology, 177(10), 1118-1127.

Condon, J. T. (1993). The premenstrual syndrome: a twin study. The British Journal of Psychiatry, 162(4), 481-486.

Delara, M., Ghofranipour, F., Azadfallah, P., Tavafian, S. S., Kazemnejad, A., y Montazeri, A. (2012). Health related quality of life among adolescents with premenstrual disorders: a cross sectional study. Health and quality of life outcomes, 10(1), 1.

Del Burgo, J. L., Trigueros, M. V., Ruiz, A. L., Muñiz, R. M., y Maestre, I. (2006). Prevalencia de síndrome disfórico premenstrual en población general. Semergen, 32(8), 367-371. https://doi.org/10.1016/S1138-3593(06)73296-8

Desai, R., Tailor, A., y Bhatt, T. (2015). Effects of yoga on brain waves and structural activation : A review. Complementary Therapies in Clinical Practice, 1-7. https://doi.org/10.1016/j.ctcp.2015.02.002

El-Lithy, A., El-Mazny, A., Sabbour, A., y El-Deeb, A. (2015). Effect of aerobic exercise on premenstrual symptoms, haematological and hormonal parameters in young women. Journal of Obstetrics and Gynaecology, 35(4), 389-392. https://doi.org/10.3109/01443615.2014.960823

Ford, O., Lethaby, A., Roberts, H., y Mol, B. (2009). Progesterone for premenstrual syndrome. Cochrane Database of Systematic Reviews, (2). 
https://doi.org/10.1002/14651858.cd003415

Frank, R. T. (1931). The Hormonal Causes of Premenstrual Tension. Archives of Neurology \& Psychiatry, 438

Gracia, C. R., Freeman, E. W., Sammel, M. D., Lin, H., Sheng, L., y Frye, C. (2009). Allopregnanolone levels before and after selective serotonin reuptake inhibitor treatment of premenstrual symptoms. Journal of clinical psychopharmacology, 29(4), 403-405.

Graze, K. K., Nee, J., y Endicott, J. (1990). Premenstrual depression predicts future major depressive disorder. Acta Psychiatrica Scandinavica, 81(2), 201-205.

Greene, R., y Dalton, K. (1953). The premenstrual syndrome. British Medical Journal, 1(4818), 10071013. https://doi.org/10.1136/bmj.1.4818.1007

Guirao-Goris, J. A., Olmedo Salas, A., y Ferrer Ferrandis, E. (2008). El artículo de revisión. Revista iberoamericana de enfermería comunitaria, 1(1), 1-25.

Hashemi, S., Tehrani, F. R., Mohammadi, N., Dovom, M. R., Torkestani, F., Simbar, M., y Azizi, F. (2016). Comparison of metabolic and hormonal profiles of women with and without premenstrual syndrome: A community based cross-sectional study. International journal of endocrinology and metabolism, 14(2).

Kendler, K. S., Silberg, J. L., Neale, M. C., Kessler, R. C., Heath, A. C., y Eaves, L. J. (1992). Genetic and environmental factors in the aetiology of menstrual, premenstrual and neurotic symptoms: a population-based twin study. Psychological Medicine, 22(1), 85-100.

Kroll-Desrosiers, A. R., Ronnenberg, A. G., Zagarins, S. E., Houghton, S. C., Takashima-Uebelhoer, B. B., y Bertone-Johnson, E. R. (2017). Recreational physical activity and premenstrual syndrome in young adult women: A cross-sectional study. PLOS ONE, 12(1), 1-13. https://doi.org/10.1371/journal.pone.0169728

Marjoribanks, J., Brown, J., O'Brien, P. M. S., y Wyatt, K. (2013). Selective serotonin reuptake inhibitors for premenstrual syndrome. Cochrane Database of Systematic Reviews, (6).

Martinez, P. E., Rubinow, D. R., Nieman, L. K., Koziol, D. E., Morrow, A. L., Schiller, C. E., ... Schmidt, P. J. (2015). 5 a-Reductase Inhibition Prevents the Luteal Phase Increase in Plasma Allopregnanolone Levels and Mitigates Symptoms in Women with Premenstrual Dysphoric Disorder. 1-10. https://doi.org/10.1038/npp.2015.246

Mohebbi Dehnavi, Z., Jafarnejad, F., y Sadeghi Goghary, S. (2018). The effect of 8weeks aerobic exercise on severity of physical symptoms of premenstrual syndrome: A clinical trial 
study. BMC Women's Health, 18(1), 1-7. https://doi.org/10.1186/s12905-018-0565-5

Naheed, B., Kuiper, J. H., Uthman, O. A., O'Mahony, F., y O'Brien, P. M. (2017). Non-contraceptive oestrogen-containing preparations for controlling symptoms of premenstrual syndrome. Cochrane Database Syst Rev, 3(3), Cd010503. https://doi.org/10.1002/14651858.CD010503.pub2

O'Brien, P. M. S., Bäckström, T., Brown, C., Dennerstein, L., Endicott, J., Epperson, C. N., Steiner, M. (2011). Towards a consensus on diagnostic criteria, measurement and trial design of the premenstrual disorders : the ISPMD Montreal consensus. Arch Womens Ment Health, 14, 13-21. https://doi.org/10.1007/s00737-010-0201-3

Pearlstein, T. B., Bachmann, G. A., Zacur, H. A., y Yonkers, K. A. (2005). Treatment of premenstrual dysphoric disorder with a new drospirenone-containing oral contraceptive formulation. Contraception, 72(6), 414-421. https://doi.org/10.1016/j.contraception.2005.08.021

Perkonigg, A., Yonkers, K. A., Pfister, H., Lieb, R., y Wittchen, H. U. (2004). Risk factors for premenstrual dysphoric disorder in a community sample of young women: the role of traumatic events and posttraumatic stress disorder. The Journal of clinical psychiatry.

Pilver, C. E., Kasl, S., Desai, R., y Levy, B. R. (2011). Health advantage for black women: patterns in pre-menstrual dysphoric disorder. Psychological medicine, 41(8), 1741-1750.

Qiao, M., Zhang, H., Liu, H., Luo, S., Wang, T., Zhang, J., y Ji, L. (2012). Prevalence of premenstrual syndrome and premenstrual dysphoric disorder in a population-based sample in China. European journal of obstetrics \& gynecology and reproductive biology, 162(1), 83-86.

Ramírez, W., Vinaccia, S., y Gustavo, R. S. (2004). El impacto de la actividad física y el deporte sobre la salud, la cognición, la socialización y el rendimiento académico: una revisión teórica. Revista de estudios sociales, (18), 67-75.

Rubinow, D. R., y Roy-Byrne, P. (1984). Premenstrual syndromes: Overview from a methodologic perspective. Am J Psychiatry, 141(2), 163-172.

Schmidt, P. J., Martinez, P. E., Nieman, L. K., Koziol, D. E., Thompson, K. D., Schenkel, L., ... y Rubinow, D. R. (2017). Premenstrual dysphoric disorder symptoms following ovarian suppression: triggered by change in ovarian steroid levels but not continuous stable levels. American Journal of Psychiatry, 174(10), 980-989.

Solís, J. C. (2013). Diagnóstico y tratamiento del síndrome premenstrual. Revista médica de costa rica y Centroamérica, 70(608), 709-715. 
Teixeira, A. L. da S., Oliveira, É. C. M. e, y Dias, M. R. C. (2013). Relação entre o nível de atividade física e a incidência da síndrome pré-menstrual. Revista Brasileira de Ginecologia e Obstetrícia, 35(5), 210-214. https://doi.org/10.1590/s0100-72032013000500004

Tsai, S. Y. (2016). Effect of yoga exercise on premenstrual symptoms among female employees in Taiwan. International Journal of Environmental Research and Public Health, 13(7). https://doi.org/10.3390/ijerph13070721

Tsai, S. Y., Kuo, F. C., Kuo, H. C., y Liao, L. L. (2018). The prevalence of self-reported premenstrual symptoms and evaluation of regular exercise with premenstrual symptoms among female employees in Taiwan. Women and Health, 58(3), 247-259. https://doi.org/10.1080/03630242.2017.1296056

Wittchen, H. U., Becker, E., Lieb, R., y Krause, P. (2002). Prevalence, incidence and stability of premenstrual dysphoric disorder in the community. Psychological medicine, 32(1), 119132.

Wu, W., Lin, T., Chu, I., y Liang, J. (2015). The Acute Effects of Yoga on Cognitive Measures for Women with Premenstrual Syndrome. 21(6), 364-369. https://doi.org/10.1089/acm.2015.0070

Wyatt, K. M., Dimmock, P. W., Ismail, K. M. K., Jones, P. W., y O'Brien, P. M. S. (2004). The effectiveness of GnRHa with and without "add-back" therapy in treating premenstrual syndrome: A meta analysis. BJOG: An International Journal of Obstetrics and Gynaecology, 111(6), 585-593. https://doi.org/10.1111/j.1471-0528.2004.00135.x

Yonkers, K. A., y Simoni, M. K. (2017). Premenstrual disorders: An expert review. American journal of obstetrics and gynecology, 218(1), 68-74.

Zhang, H., Zhu, M., Song, Y., y Kong, M. (2015). Baduanjin exercise improved premenstrual syndrome symptoms in Macau women. Journal of Traditional Chinese Medicine, 34(4), 460-464. https://doi.org/10.1016/s0254-6272(15)30047-9 


\section{Anexos}

Anexo I. Ejemplo de sesión.

\section{EJEMPLO DE SESIONN DIARIA DE YOGA}

MATERIALES: Una esterilla por persona.

\section{CALENTAMIENTO}

TIEMPO

Ejercicios respiratorios (Pranayama)

$5^{\prime}$

Bhramari

- Se inicia con una posición padmasna, sidhasana o valjrasana.

- A continuación, se cierran los oídos y los ojos con las puntas de los pulgares y los dedos restantes, respectivamente. Es necesario aplicar un poco de presión para aislarse de la luz y el sonido exterior.

- El dedo índice debe colocarse a lo largo del

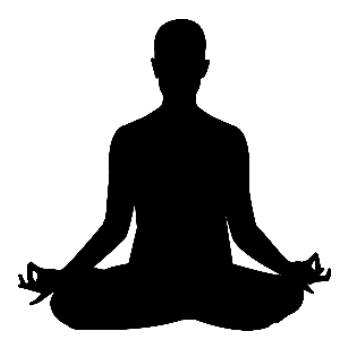
hueso de la ceja y son los otros dos dedos los que cubren ligeramente los ojos.

- Tras aislarnos del exterior, se realiza una inhalación profunda.

- Se exhala lentamente, soltando el aire por la nariz todo el tiempo. Es necesario sentir las vibraciones que produce esta exhalación.

El ejercicio se repite 5 veces y se termina con tres respiraciones normales antes de liberar los oídos y los ojos. 


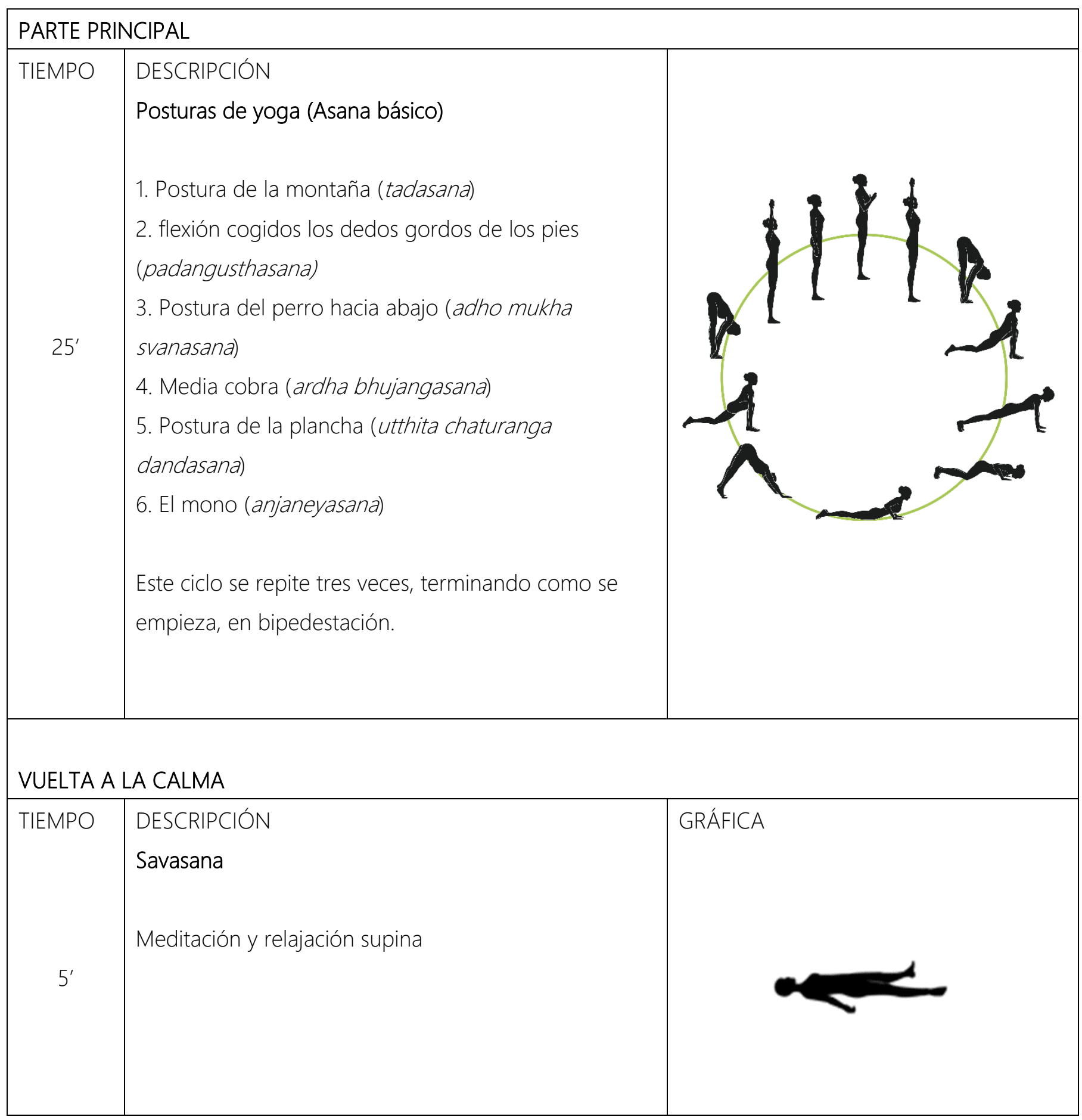

